\title{
No Reference Without Referents ${ }^{1}$
}

\section{(Sin referentes no hay referencia)}

\author{
Eduardo GARCÍA RAMÍREZ
}

Recibido: 10 de junio de 2014

Aceptado: 11 de marzo de 2015

\begin{abstract}
Sainsbury (2005) and (2009) offers a theory of empty names that purports to account for the content and truth-value of all utterances involving them. The goal is to do this while offering a homogenous semantic treatment: both empty and nonempty names make the same kind of contribution to truth-values. The account is based on a new theory of reference that purports to be an alternative among nondescriptivist accounts. According to the new theory, there is reference even without referents. In this paper I argue that the theory does not offer a homogeneous semantics for names and that, thus, it fails to offer a more satisfactory alternative to the theories already available. I conclude by briefly describing a way in which a theory could in fact offer such homogeneous semantics for names via a cognitive theory of empty names.
\end{abstract}

Keywords: homogeneous semantics, truth conditions, cognitivism.

\section{Desiderata}

Reimer (2001a) identifies two problems related to the ordinary use of empty names. On the one hand, speaker intuitions support the idea that statements using empty names are meaningful and truth-evaluable. Reimer dubs this "the intuition

\footnotetext{
1 The author would like to thank an anonymous referee for his/her helpful comments. The research for this paper was supported by PAPIIT-IN400915 and CONACYT CCB-2011-166502 research projects. The paper was completed as a visiting scholar at the UBA, Buenos Aires, Argentina. Thanks are owed to CONACYT (Of. 233188) and DGAPA (UNAM), PASPA program, for granting the needed scholarships.
} 
problem." On the other hand, speaker behavior suggests that there is something that they affirmed, denied, etc. while using empty names. As theorists, we are left with the task of describing some proposition or other that is asserted, denied, etc. Reimer calls this "the proposition problem."

I believe there is more to this story. Speaker behavior points to many other problematic tasks left for us to work out. Competent speakers do not only use empty names to assert simple declarative statements; they also use empty names to engage in games of make-believe, and to make belief reports that describe behavior adequately both, within and without games of make-believe. The use of empty names within games of make-believe demands an account that is consistent with the fact that competent speakers may use empty names to say something true. Adequate belief reports that make use of empty names suggest that the proposition expressed by the embedded that clause has all the explanatory virtues of a singular proposition. A satisfactory account of empty names must solve the "intuition" and "proposition" problems in a way that is fitting for attitude ascriptions and fictional uses of empty names.

Given these constraints there are three desiderata that any satisfactory account of empty names should endeavor to satisfy:

(i) A semantic treatment of empty names that yields a solution to Reimer's 2001 a problems;

(ii) An account that fits well with the explanatory success of belief reports using empty names; and

(iii) An account that is at least consistent with the fictional use of empty names.

\section{The theory and its success}

Sainsbury (2005) and (2009) presents a theory meant to solve these problems by defending the following central claims (see Sainsbury 2005, p. 45-46):

$\mathrm{RWR}_{1}$ : All referential expressions, and particularly names (empty or not), are semantically homogenous. They all have the same semantic behavior and determine truth conditions in the same way.

$\mathrm{RWR}_{2}$ : All names are associated with referential conditions of the form:

$\forall \mathrm{x}\left({ }^{\prime} \mathrm{N}_{\mathrm{R}} \mathrm{x} \equiv \mathrm{x}=\mathrm{n}\right) \quad$ where 'XRY' takes $\mathrm{X}$ to refer to $\mathrm{Y}$.

$\mathrm{RWR}_{3}$ : 'S is p' is false if and only if either (i) ' $\mathrm{S}$ ' lacks a referent; or (ii) ' $\mathrm{S}$ ' ' $\mathrm{S}$ referent lacks the property referred to by ' $p$ '.

$\mathrm{RWR}_{4}$ : All names are rigid designators. 
$\mathrm{RWR}_{5}$ : Not all individual constants are assigned a referent and empty domains may be relevant for the interpretation, even in cases of validity (i.e., Negative Free Logic is endorsed).

Together $\mathrm{RWR}_{1}$ and $\mathrm{RWR}_{2}$ entail that all names, empty or not, have a meaning: i.e., the referential condition described by $\mathrm{RWR}_{2}$. There can be, on this view, reference without referents (hence the label "RWR"). Non-empty names will have a referent on top of this. This allows us to understand the intelligibility of empty names. According to this view, an assertion of ' $\mathrm{N}$ is $\mathrm{F}$ ', where ' $\mathrm{N}$ ' is an empty name and ' $\mathrm{F}$ ' is a predicate, will be understood in terms of something like:

$$
\forall \mathrm{x}\left({ }^{\prime} \mathrm{N}{ }_{\mathrm{R}} \mathrm{x} \equiv \mathrm{x}=\mathrm{n} \wedge \mathrm{Fx}\right)
$$

This helps illuminate our first desideratum: the "proposition" problem. The content of a sentence using an empty name is modeled by the quantified first order logic sentence above. According to $\mathrm{RWR}_{3}$ all affirmative sentences using empty names are false. So this solves the "intuition problem." Competent speakers think that assertions using empty names are truth-evaluable because all assertions using empty names are truth-evaluable. All affirmative ones are, in fact, false. Sainsbury thinks this also delivers an account of how negative existential assertions may express truths. For ' $\mathrm{S}$ doesn't exist' just is the negation of ' $\mathrm{S}$ exists.' If ' $\mathrm{S}$ ' is empty, then ' $\mathrm{S}$ exists' is false and, hence, its negation (i.e., ' $\mathrm{S}$ doesn't exist') is true.

Sainsbury's account seems also capable of accounting for the second desideratum: the "belief reports" problem. Sally leaves cookies and milk by the Christmas tree and prepares herself to stay up all night. How should we describe her mental states? It seems natural to claim the following: "Sally believes that Santa is coming tonight." According to Sainsbury's (2005) account, the embedded sentence in this belief report is understood as conveying something like the following first-order logic sentence:

\section{$\forall \mathrm{x}\left(\right.$ 'Santa' $_{\mathrm{R}} \mathrm{x} \equiv \mathrm{x}=$ SANTA $\left.\wedge \mathrm{Cx}\right)$ \\ (where $\mathrm{Cx}$ : $\mathrm{x}$ is coming tonight)}

This helps illuminate the explanatory role of belief reports because it offers a truth-evaluable content that is sensitive to changes in the use of different empty names, since the reference conditions are, as Sainsbury puts it, homophonic - i.e., they mention and use the very name they define in the right hand side. If we can distinguish between 'Hamlet' and 'Santa' we can distinguish between claims about Hamlet and claims about Santa. Hence, we can also distinguish between beliefs 
about the former and beliefs about the latter. Thus, Sainsbury's (2005) account can satisfy the second desideratum.

Sainsbury (2009) also offers an account of the third desideratum: the fictional use of empty names. On his view, we may sometimes have to appeal to (possibly implicit) fiction operators in order to explain the truth-values associated to the use of fictional names. This operator is a shorthand for the disclaimer "in the fictional story it is the case that [...]." Such operator would explain why, even though they are all false, we take declarative utterances involving empty names to be true. What speakers say, according to Sainsbury, is that their utterances are true in the fiction, and this might be the case even if they are in fact false. Briefly put, that is how RWR deals with the "fiction problem." Fictional uses come with a fiction operator.

At this point one wonders: what role does $\mathrm{RWR}_{5}$ play in this whole story? I will address this question in what follows. But first, let me compare RWR against three alternative non-descriptivist accounts of empty names.

\section{RWR and other theories of empty names}

There are at least two other theories in the literature that offer an account of the desiderata (i)-(iii).

On the one hand, there is Reimer's (2001b) "Meinongian" theory. On her view, all names convey their referent. Since they lack one, empty names do not convey any meaning. Thus, assertions using empty names are, strictly speaking, meaningless and not truth-evaluable. Yet, argues Reimer (2001b), competent speakers are unconscious Meinongians that unreflectively assume that all names, including empty ones, have a referent. It is not difficult to show how this "Meinongian" assumption ultimately accounts for (i)-(iii). The proposition problem is weakened and solved by claiming that the relevant content is a proposition with a gap in place of the lacking referent. The intuition problem is solved directly: speakers think they are conveying useful information because they are unconscious Meinongians. For the same reason, speakers think the assertions are truth-evaluable. Even more, Reimer can also claim that this unconscious Meinongianism can guide speaker's belief formation and, thus, explain the success of belief reports using empty names. Speaker beliefs about fictional uses of names could be explained in a similar fashion. Reimer's theory accounts for all the desiderata, but it does so by assuming that speakers are unreflective Meinongians. This assumption is not only controversial and somewhat tendentious, it also seems implausible and there is no independent evidence on its behalf.

On the other hand, there are explicitly meinongian views, such as the one developed by Salmon (1998) and followed by Soames (2002). On this view, most empty 
names are not really empty, they have a referent such as a fictional object. Such a theory very clearly offers a homogeneous semantic account of names while explaining the desiderata in a very direct way. Yet, the assumption that there exist fictional objects seems implausible, not so parsimonious, and appears to have no independent evidence on its behalf.

Both these views work out well with a homogeneous semantics for names. But the cost in plausibility and parsimony seems high. Against this background, Braun (1993) and (2005) offers yet another non-descriptivist account. This view has the virtue of not carrying implausible requirements while remaining parsimonious. Like Reimer (2001b), it claims assertions with empty names express propositions with a gap in place of the lacking referent. Unlike Reimer (2001b), it does not take speakers to be unreflective Meinongians. Instead, Braun offers a heterogeneous semantics for names. Declarative assertions are true if the referent of the name bears the property referred to by the predicate, in all other cases they are false. Since assertions involving empty names do not convey a referent, they fall under the "other cases" label and determine truth-conditions in their own way: they are all false.

Against these alternatives, RWR seems like the best option: it offers a theory with no implausible requirements while purporting to have a homogeneous semantic treatment: all names, empty or not, semantically convey a referential condition. I will argue, however, that this latter requirement is never met by RWR. It is true that according to RWR 2 all names have the same semantic content. However, as I will show in what follows, this thesis plays no role when it comes to explaining how empty names determine truth-conditions. To do this Sainsbury must give up the chances of offering a homogeneous semantics for names.

\section{Negative Free Logic and truth conditions in RWR}

It is remarkable that RWR offers a way to account for all the desiderata. Sainsbury (2005) explicitly claims that for this to be the case one must depart from Classical Logic and endorse what Burge (1974) calls "Negative Free Logic" (NFL).

To endorse NFL (see Sainsbury 2005, Chapter 2) one must be prepared to accept that: (1) individual constants may be assigned no entity from the domain of the interpretation function; and (2) that empty domains may be relevant, even for cases of validity. This is a substantial departure from Classical Logic. I will not try to show how problematic the endorsement of (1) and (2) may be. ${ }^{2}$ It will be enough to make clear that this move plays a central role in RWR.

\footnotetext{
2 There are in fact several problems associated with free logics in general and with negative free logic in particular. For more on this see Nolt (2010).
} 
Consider how RWR looks like without $\mathrm{RWR}_{5}$. All we have is $\mathrm{RWR}_{1}, \mathrm{RWR}_{2}$, and $\mathrm{RWR}_{4}$, since NFL is required for $\mathrm{RWR}_{3}$.

$\mathrm{RWR}_{1}$ : All referential expressions, and particularly names (empty or not), are semantically homogenous. They all have the same semantic behavior and determine truth conditions in the same way.

$\mathrm{RWR}_{2}$ : All names are associated with referential conditions of the form:

$\forall \mathrm{x}\left({ }^{\prime} \mathrm{N}_{\mathrm{R}} \mathrm{x} \equiv \mathrm{x}=\mathrm{n}\right) \quad$ where 'XRY' takes $\mathrm{X}$ to refer to $\mathrm{Y}$.

$\mathrm{RWR}_{4}$ : All names are rigid designators.

In particular, the assignment of truth-values described in $\mathrm{RWR}_{3}$ is incompatible with Classical Logic (CL).

$R_{2}$ : ' $S$ is p' is false if and only if either (i) ' $S$ ' lacks a referent; or (ii) ' $S$ ' ' $S$ referent lacks the property referred to by ' $\mathrm{p}$ '.

In cases where there is no referent, there is no assignment of truth-values according to CL. Thus, without $\mathrm{RWR}_{5}$ we cannot get the assignment described in $\mathrm{RWR}_{3}$-(i).

What follows from this is quite simple. Even though it offers an account of the content of empty names, $\mathrm{RWR}_{2}$ gives at best a partial explanation. Without NFL and, thus, without $\mathrm{RWR}_{3}$-(i), RWR is unable to explain how sentences using empty names can be truth-evaluable. It is, of course, not surprising that a proper part of the theory fails to achieve everything the whole theory is meant to achieve. But it is worth noting that the only part of the theory that may achieve the theory's goal of homogeneity fails to do so. As I will show in the following section, $\mathrm{RWR}_{3}$ and $\mathrm{RWR}_{4}$ entail that the theory offers heterogeneous truth conditions for empty and non-empty names. It seems, as I will argue, that it is this heterogeneous element of RWR that accounts for desideratum (i): that ordinary predications using empty names are (or seem to be) truth-evaluable and that negative existentials using empty names are true.

It is clear, then, that without NFL Sainsbury's novel theory of reference without referents cannot achieve what it is supposed to achieve. The intelligibility of empty names requires an explanation of their truth-evaluability. This explanation, in the case of RWR, comes solely from the endorsement of NFL and, with it, of $\mathrm{RWR}_{3}$ (i).

It seems clear, then that NFL allows RWR to claim what those who do not endorse NFL cannot: that all names have the same kind of meaning -e.g., a referential condition- while still offering truth conditions for assertions involving empty names. This is already a bad result for RWR, for it shows that the novel claim that there can be reference without referents in virtue of there being a referential condi- 
tion as part of the semantics for names plays a dispensable explanatory role. They assign semantic properties that allow empty names to be used to express truths. But all this can be done by merely keeping NFL and letting the new theory of reference go. This way one can still get an account of the truth-evaluability and, hence, intelligibility of empty names as witnessed by Braun's (1993) and (2005) proposal. The inverse, however, is not possible; keeping the new theory of reference without NFL will not explain what must be explained.

\section{Heterogeneous truth conditions}

I think RWR $R_{1}$ is not so obviously true in the light of $\mathrm{RWR}_{3}$ and $\mathrm{RWR}_{4}$. According to $\mathrm{RWR}_{4}$ non-empty names have a referent in all possible worlds. Sainsbury (2005) agrees that there is no such thing as the referent of an empty name. They do not refer to nonexistent objects, fictional characters, or merely possible individuals on his view. Thus, it also follows from $\mathrm{RWR}_{4}$ that empty names lack a referent in all possible worlds.

According to $\mathrm{RWR}_{3}$ there are two semantically determined rules for assigning truth conditions. These in turn depend on whether or not the name has a referent. First, if the name has a referent, truth depends on the object's properties. Second, if the name lacks a referent, the sentence is immediately false. These are two clearly distinct semantically determined rules for truth-condition determination and so, presumably, two clearly distinct kinds of semantic behavior. The very fact that the presence or lack of a referent determines how truth values are fixed, makes it so that empty names and non-empty names make different contributions to truth-values in all possible worlds.

Non-empty names have a referent in all worlds. Thus, they determine truth conditions in the first way: the truth of the relevant non-negated sentence always depends on the properties of the relevant object. Empty names, however, lack a referent in all worlds. Hence, they determine truth conditions in the second way: they always determine the relevant non-negated sentence to be false in virtue of lacking a referent. From this it follows that empty names and non-empty names do have an important semantic difference: they do determine truth conditions in different ways.

It is important to note that the semantic difference entailed by $\mathrm{RWR}_{3}$ and $\mathrm{RWR}_{4}$ is not a mere difference in truth conditions, such as the difference there might be between 'Smith' and 'Jones' in virtue of the fact that one appeals to Smith and the other to Jones in order to determine truth conditions. The difference is substantially semantic in virtue of the fact that it is a difference between the semantically determined rules that, according to $\mathrm{RWR}_{3}$, determine the truth of sentences using empty and non-empty names. Thus, it is more like saying that 'Smith' and 'Jones' 
are semantically heterogeneous since the truth of sentences involving 'Smith' always depend on Smith while sentences involving 'Jones' are always false. That is what $\mathrm{RWR}_{3}$ and $\mathrm{RWR}_{4}$ entail concerning empty and non-empty names.

I am not claiming that it is merely in virtue of the fact that empty names lack referents in all worlds while non-empty names have them in all worlds that RWR offers a heterogeneous semantics. It is in virtue of this together with Sainsbury's further claim (see $\mathrm{RWR}_{3}$ ) that whether the name has or lacks a referent is relevant to pick the rule that will determine how the name will contribute to truth conditions. This claim puts the presence or absence of referents right into the semantic determination of truth conditions. Whether or not the speaker knows whether the name she is using is empty or not is irrelevant here. The theory itself, which is a theory of the semantics of empty and non-empty names, makes the referential distinction semantically relevant.

Sainsbury (2005) claims that RWR offers a homogeneous semantics for empty and non-empty names because both are supposed to get axioms of the same form (i.e., Sainsbury's homophonic reference condition). This is, certainly, an element of homogeneity among them. But is that enough to claim they are semantically homogeneous? As I have shown empty and non-empty names end up determining truth conditions in different semantically determined ways, they make different contributions to truth-values in all cases. If a common reference condition is enough for homogeneity, varied contributions to truth-values should be enough for heterogeneity. Hence, it is far from clear that RWR offers a homogeneous semantic account for empty and non-empty names.

Compare RWR with an explicitly heterogeneous semantics for empty and nonempty names. On this view, non-empty names have their referent as semantic content and contribute to truth-values in the first way: i.e., the relevant declarative assertion is true if and only if the referent has the property in question. On this view, non-empty names do not contribute anything like a reference condition. Empty names, however, are a different thing. They do not contribute their referent as semantic content. Instead what they have is a function mapping all relevant properties into the truth-value false. Therefore, empty names contribute to truth-values always in the second way: i.e., all declarative assertions involving them are ipso facto false. On this view, empty names also do not contribute anything like a reference condition. This theory will always predict exactly the same truth-values as RWR would for declarative assertions using either empty or non-empty names. Yet, the theory is clearly heterogeneous.

To illustrate this point further consider the following substantial semantic difference between empty and non-empty names. Given $\mathrm{RWR}_{3}$ and $\mathrm{RWR}_{4}$ it is possible to find declarative assertions that would be contradictory when using non-empty names that, nonetheless, turn out to have the same truth-value in the same context 
when using empty names. Such is the case of 'Hamlet is tall' and 'Hamlet is short' in a non-fictional context-when speakers are not taken to be speaking within a fiction. Both assertions are false. This phenomenon, however, is not possible with nonempty names according to $\mathrm{RWR}_{3}$ and $\mathrm{RWR}_{4}$. There is no single context at which 'Mark Sainsbury is tall' and 'Mark Sainsbury is short' have the same truth-value?.

It seems that this is a serious problem for RWR. Exactly how serious it is depends on the theory's goal. As long as $\mathrm{RWR}_{1}$ remains a central motivating thesis, the objection proves to be substantial. There are reasons to think that $\mathrm{RWR}_{1}$ is a central part of Sainsbury's account of empty names. Sainsbury (2005) offers it as one of RWR's distinctive and most attractive features. It provides a good reason to think that RWR is superior to most accounts of empty names in the literature. Furthermore, if RWR does not intend to offer such homogenous semantics for names, then it is unclear why one should accept the theory as a whole together with its endorsement of NFL. After all, satisfactory heterogeneous treatments of empty names are already available for those who do not want to accept that there can be reference without referents.

\section{Homogeneity?}

Sainsbury could reply to the objection of heterogeneity (see section 4) by distinguishing between what the users of empty and non-empty names may know and what the actual mechanism that determines truth-values may be. ${ }^{4}$ Users of names need not know that the name they are using is empty, and even if they do so, or if they change their mind about the names they use, there need not be a change in meaning between empty and non-empty names. For all users know, this defense of RWR says, every use of a name, whether empty or not, conveys the reference criterion described by $\mathrm{RWR}_{2}$.

$\mathrm{RWR}_{2}$ : All names are associated with referential conditions of the form:

$\forall \mathrm{x}\left({ }^{\prime} \mathrm{N}{ }_{\mathrm{R}} \mathrm{x} \equiv \mathrm{x}=\mathrm{n}\right)$ where 'XRY' takes $\mathrm{X}$ to refer to $\mathrm{Y}$.

Notice, however, that this reply requires a split between knowledge of use and the determination of truth-conditions. The former may well be theoretically relevant, but it is uncontroversial that the latter is the semantically relevant one. One

\footnotetext{
3 I am aware of the fact that 'Mark is tall' and 'Mark is short' can both be used to say something true provided we change the standards of measurement. But there is no way to do this without changing the context. So it is still the case that 'Mark is tall' and 'Mark is short' cannot both have the same truthvalue in the same context.

${ }^{4}$ Mark Sainsbury has described this response in personal communication.
} 
can easily add to the above defense of RWR that, for all that truth conditions care, empty names and non-empty names are not homogeneous. Thus, we have two levels, one at which empty and non-empty names have a homogenous treatment and one at which they do not. The central question is whether this is really a homogeneous semantics for names.

It seems clear that the homogeneity does not lie at the truth-conditional level. At best it could be part of a truth-conditionally irrelevant semantic level. Perhaps there is such semantically superficial level, as Sainsbury suggests (pers. comm.). The tradition has consistently considered such aspects of language use - i.e., those that do not determine truth-conditions, to be pragmatic precisely in virtue of their truth-conditional irrelevance. Since Frege's (1892) sense-reference distinction all complex semantic distinctions, including Kaplan's (1989) character, Lewis' (1980) index, and what not, are deemed to be truth-conditionally relevant in precisely the same way that Sainsbury's reference condition is not. Furthermore, all of the so called "reference theories of meaning" (see Speaks 2014) are explicitly aiming at truth conditions. This makes Sainsbury's proposal even more odd. It is supposed to be an account of reference determination that, surprisingly, ends up being not only insufficient to fix truth-conditions, even worse, it is irrelevant to do so. Frege's senses, Kaplan's character, Lewis' index, all of them are truth-conditionally relevant and, hence, they are part of a proposed semantics.

Thus, it seems that the only reason to think that the $\mathrm{RWR}_{2}$ is part of the semantics is that in doing so Sainsbury can claim to have a level of semantic homogeneity. But that just seems like an unsuccessful way to argue for the semantic homogeneity between empty and non-empty names. This should be enough to show that RWR's homogeneity is not as complete and transparent as Sainsbury seems to think. There are other truly homogeneous accounts (see for example Reimer 2001a and 2001b, and Salmon 1998) that leave RWR wanting of homogeneity.

There are further problems with the resulting proposal. Suppose, for the sake of argument, that we admit truth-conditionally irrelevant conditions to be part of the semantics. This opens up a question: is this truth-conditionally irrelevant homogeneity enough to justify the acceptance of NFL (see section 3) and to make of RWR an outstanding theory of empty names? The answer, as I hope to show in the following and final section, is negative. One can have a truly semantically homogeneous account of empty names that gives us all we want without departing from Classical Logic.

It seems fair to say, then, that RWR has failed to offer what it promised: a truly homogeneous semantic treatment for empty and non-empty names. 


\section{An alternative account of empty names}

Sainsbury (2005) and (2009) offers a new theory of empty names based on a new theory of reference: the theory of reference without referents or RWR. This theory promises to offer a homogeneous semantics for names that meets all the desiderata involved in the problem of empty names. Crane (2008), Dumitru and Kroon (2008), García-Carpintero (2008), and Orlando (2008) have offered different objections against Sainsbury's account, all of which have been properly accounted for (see Sainsbury 2008). In this paper I have presented a new objection that has not been considered by Sainsbury's opponents. I have argued that RWR does not offer a truly homogenous semantics for names and, hence, that it fails to be the novel and satisfactory theory it purports to be. In so doing, I have shown that what really does the explanatory work within Sainsbury's theory is his endorsement of NFL together with heterogeneous truth-conditions that distinguish between empty and nonempty names. This suggests that Sainsbury's novel view on reference, according to which there can be reference without referents, is not as useful a theory of empty names as it claims to be.

It seems as if non-descriptivists will have a difficult time finding a plausible and parsimonious theory of empty names that may still offer a homogeneous semantics. It may also seem that I have set up the bar too high for RWR by asking it to deliver where no one can. This appearance, however, is mistaken. To my mind there is a way out of this conundrum: a plausible and parsimonious theory of empty names paired with a homogeneous semantic treatment for names. In what follows I will present a brief account of such a theory hoping to say enough to convince the reader (for a full and detailed version of the account, see García-Ramírez 2011).

Judging by the literature, the only way to have a parsimonious theory with a homogenous semantics consists in offering a non-semantic explanation of the meaningfulness and truth-evaluability intuitions of competent speakers. Braun (2005), Sainsbury (2005) and Salmon (1998) fail to offer such an account precisely because they want to explain these intuitions in semantic terms. Braun and Sainsbury end up having a heterogeneous semantics and Salmon keeps homogeneity at a very high ontological price. Reimer (2001b) is an exception here. However, her non-semantic account consists of an implausible and unsupported appeal to unconscious Meinongianism among competent speakers.

A better theory will result from a plausible non-semantic account of the truth value and meaningfulness intuitions that competent speakers have with respect to ordinary uses of empty names. One way of gaining plausibility consists in offering an explanation that has some independent support. I believe some such account is forthcoming from the research on pretense abilities done by cognitive psychologists. Most experts on pretense (see Leslie 1987 and Nichols and Stich 2000) agree 
that pretense involves a general ability to manipulate and interpret representations. This cognitive ability is certainly not linguistic and, hence, its resources are not semantic. This suggests that a cognitive theory of empty names can deliver a plausible non-semantic account of the truth value and meaningfulness intuitions of competent speakers. All we need to do is pay attention to the cognitive mechanisms involved in pretense to see how they can explain the way in which speakers interpret representations by means of pretense cognitive abilities.

Such a cognitive, non-descriptive, theory of empty names would depend essentially on the following thesis:

Cognitivism: Serious assertions, belief reports, and fictional uses of empty names are meaningful and intelligible mainly in virtue of the cognitive-general resources they recruit and not in virtue of their semantics alone. Such resources are triggered by the subject's perceptual system. Whether or not the subject knows (or believes) the name to be empty need not trigger the use of such resources.

One can safely expect this theory to be plausible. At the very least, it will be as plausible as the cognitive psychological theories of pretense that psychologists may offer based on their empirical research. Such a theory would also be semantically homogeneous, for the explanatory burden will lie mainly upon the cognitive account. Speaker intuitions, belief formation, and fictional uses of empty names may also be explained by the existence of such cognitive mechanism. All we need is some independent evidence on behalf of the existence of such mechanism. We can borrow this from cognitive psychology.

Here is how the account works. ${ }^{5}$ Start from semantics. All names, whether empty or not, determine truth-conditions in exactly the same way, that is, by contributing a referent. When names are empty, there is no contribution. If there is no referent there just is nothing left semantically speaking. Contrary to Sainsbury's dictum, there is no reference without referents. Hence, there is nothing to be evaluated for its truth. Strictly speaking, nothing that may be said by using empty names can in any sense be true or false. It is in fact truth-valueless. If you prefer, we can say that all linguistic practice involving referential uses of empty names say something false in virtue of the above.

But speakers have strong intuitions to the point that they can say true things, make accurate belief ascriptions, by using empty names referentially. How is that possible? According to Leslie (1998) and Nichols and Stich (2000), we humans are able to process representations in a way that allows us to manipulate such representations. Leslie specifically posits something like a decoupler, a mechanism that copies, interprets, and manipulates representations for all sorts of purposes. To illus-

5 For a detailed version of the account see García-Ramírez (2011). 
trate, consider a five year-old that is perceptually aware of a telephone and, hence, has formed the representation "this is a telephone". According to Leslie, for this five year-old to use, in pretense play, a banana as if it were a telephone, she must take the representation "this is a telephone" and copy it (by divorcing it from its content but not from its structure), then reassign contents (by substituting the telephone for a banana as the referent of 'this') and deliver the resulting representation to central cognition. All this allows the child to use the representation "this is a telephone" to pretend that the banana is a telephone.

The cognitive theory of empty names offers some such account of what goes on with the use of empty names. It claims that whenever there is no referent available, either in perception or in memory, speakers are forced to reinterpret the relevant representation. Such need triggers something like Leslie's decoupler mechanism, allowing the speaker to reinterpret the relevant representation by assigning whichever referent it may turn out to be useful in the context. This delivers a meaningful representation, with an assigned object. This representation may seem to be evaluated for truth and meaningfulness. Thus explaining the intuitions of speakers.

The account, however, is not semantic. This specifically means that the assignment of an object to interpret the representation is not done by the semantics but by an extra-linguistic mechanism. This, in turn, means that the object is not in any sense a referent. Any object will do to interpret the representation. It is better to understand the object as playing a role of object substitution. The assigned object is there just to substitute the original referent which, of course, is not available.

Another important feature of this account of speaker intuitions is that it is not meant to work at a personal level. The view does not claim or require that speakers be consciously aware of the difference between a manipulated representation and one that is fully determined semantically. For all the speaker knows, there might be no difference between a representation using empty names and one using nonempty names.

This latter point is important because it allows for the cognitive account to differ from what have been known as pretense accounts of empty names. The account is not that speakers intuitively believe that sentences using empty names are true because they imaginatively engage in a pretense where the relevant sentences are true in virtue of the speaker's make believe. Whether or not the speaker is engaged in some sort of pretense or game of make believe is absolutely irrelevant to this account. What triggers the use of the cognitive mechanisms that allow speakers to manipulate representations and assign objects to empty names is, on this view, the absence in perception and memory of anything like a semantically assigned referent of the relevant name. This differs completely from pretense accounts. According to the latter, what explains speaker intuitions is the fact that speakers are pretending when using them. Nothing like that is going on in the cognitive account. 
Speakers need not consciously pretend in order for them to use their pretense cognitive abilities to properly engage in linguistic practice. To further illustrate how the cognitive account works, consider the following objection. The sentences (1) and (2) are intuitively true.

(1) In Doyle's Holmes novels, Sherlock Holmes solves every crime he investigates.

(2) In Doyle's Holmes novels, London is located in England.

Now consider what happens when we get rid of the fiction-inducing operator 'In Doyle's Holmes novels', as in (3) and (4)

(3) Sherlock Holmes solves every crime he investigates.

(4) London is located in England.

It seems that whatever form of pretense involved in (1) and (2) remains in (3) but not in (4). We can, for example, claim that (3) is still true in virtue of the fiction, but we cannot say the same of (4). What is puzzling here is that the presence or absence of the fiction-inducing operator seems to make a difference, given the fact that 'London' and 'Sherlock Holmes' are to be considered semantically homogenous. It seems obvious that (4) is true in virtue of the properties and relations that the referent of 'London' has or stands in. The same cannot be said of (3). If it is true, it is so in virtue of the relevant make-believe, the novel, or whatever.

This would be a serious objection if the cognitive account were to accept that (3) and (4) are true in the same sense. Fortunately, that is exactly the opposite to what the theory claims. Whoever claims that (3) and (4) are both true is equivocating between being true and there being a pretense according to which the sentence is true. Strictly speaking, in virtue of the semantics (3) is truth-valueless. And that is all there is to be said about the semantics of (3). In the same sense, (4) is true, because the very same semantics that applies to (3) applies to (4), yet (4) has a nonempty name, so it gets a truth-value. So it does not follow that 'London' and 'Sherlock' have different semantics in virtue of determining truth-conditions in different ways. They both do it the same way, by means of reference.

This, of course, leaves an open question: what is the difference then between (3) and (4) when compared with (1) and (2)? The cognitive account comes equipped with a straightforward answer. The difference is that 'London', unlike 'Sherlock', has an assigned referent such that any competent speaker will either be able to access it perceptually or by memory. Such difference makes a big difference, since 'London', unlike 'Sherlock', does not trigger the use of Leslie's representation decoupling mechanism. So 'London' gets an interpretation merely in virtue of its 
semantics. In other words, having or lacking a referent makes up for a big cognitive difference, but not for a semantic one. Empty and non-empty names remain, semantically speaking, homogenous. They all make exactly the same semantic contribution to truth-conditions, that is, a referent. If there is no referent, then there is no semantic contribution.

\section{Final remarks}

In recent work, Mark Sainsbury has championed a so called "new theory of reference" according to which all names, whether empty or not, come semantically equipped with a reference criterion that makes all names semantically homogenous. This criterion does not guarantee that there is a referent, yet it guarantees that there is something like reference.

In this paper I have shown that this way of presenting Sainsbury's theory is misguiding. It suggests that there is substantial semantic homogeny between empty and non-empty names where there is no such thing. I have argued that Sainsbury's account works mainly on top of the acceptance of Negative Free Logic and the claim that expressions using empty names really do not determine truth-conditions in the same way as those using non-empty names. The homogeneity between empty and non-empty names is, at best, superficial and non-semantic. This, in turn, makes the acceptance of negative free logic rather expensive theoretically speaking.

In the final section I have argued that there are alternative, cognitive, ways of keeping a homogenous semantics for names, empty or not, that does not require either postulating fictional entities nor the acceptance of negative free logic. In doing so, I hope to have pointed at directions in which our understanding of empty names can be improved. I have, of course, not presented the details of the alternative theory of empty names (see García-Ramírez 2011). But I hope to have sketched some such account by suggesting that the difference between empty and non-empty names is not semantic, or even linguistic, but cognitive.

\section{Bibliographics references}

Braun, D. (1993): “Empty names." Nous, 27, 449-69

Braun, D. (2005): "Empty names, fictional names, mythical names". Nous, 39:4, 596-631

Burge, T. (1974): "Truth and singular terms." Nous, 8, 309-25.

CRANE, T. (2008): "Sainsbury on thinking about an object." Crítica 40, 120, 85-95. Dumitru, M., and Kroon, F. (2008): "What to say when there is nothing to talk about." Crítica 40, 120, 97-109. 
Frege, G. (1892): "On Sense and Reference", in Translations from the Philosophical Writings of Gottlob Frege, P. Geach, and M. Black, (eds.) Oxford: Basil Blackwell.

García-CARPintero, M. (2008): "Homophonic prejudices." Crítica 40, 120, 67-84.

García-Ramírez, E. (2011): "A cognitive theory of empty names", Review of Philosophy and Psychology, 2, 785-807.

Kaplan, D. (1989): "Demonstratives", in Themes from Kaplan, Almong, J., Perry, J., and Wettstein, H., (eds.) Oxford: Oxford University Press.

LESLIE, A.M. (1987): "Pretense and representation: the origins of "theory of mind"." Psychological Review 94, 412-26.

LeSLIE, A.M. (1994): "Pretending and believing: issues in the theory of ToMM." Cognition 50, 211-38.

Lewis, D. (1980): "Index, Context, and Content", in Philosophy and Grammar, Kanger, S., Ohman, S., (eds.) Boston: Reidl.

Nichols, S. and Stich. S. (2000): "A cognitive theory of pretense." Cognition 74, $115-47$.

Nolt, J. (2010): "Free logic." In Stanford Encyclopedia of Philosophy, http://plato.stanford.edu/entries/logic-free/\#4

ORLANDO, E. (2008): "Fictional names without fictional objects." Crítica 40, 120, 111-127.

Reimer, M. (2001a): "The problem of empty names." Australasian Journal of Philosophy 79, 4, 491-506.

Reimer, M. (2001b): "A "Meinongian" solution to a Millian Problem." American Philosophical Quarterly 38, 3.

SAINSBURY R.M. (2005): Reference Without Referents. Oxford: Oxford University Press.

SAINSBuRY, R.M. (2008): "Intensional transitives and presuppositions." Crítica 40, $120,129-139$.

SAInSBURY, R.M. (2009): Fiction and Fictionalism. Oxford: Routledge.

SAlmon, N. (1998): "Nonexistence." Nous, 32:3, 277-319.

SoAmes, S. (2002): Beyond Rigidity. Oxford: Oxford University Press.

Eduardo García Ramírez

Instituto de Investigaciones Filosóficas

Universidad Nacional Autónoma de México

edu@filosoficas.unam.mx 